\title{
Intramedullary abscess of the cervical spinal cord in an HIV-I infected patient
}

\begin{abstract}
Intramedullary spinal cord abscess (ISCA) is a rare condition. Wedescribe the magneticresonance imaging (MRI) findings, including the diffusion-weighted imaging (DWI) findings, of a cervical ISCA in a HIV-infected 43-year-old male with breakdown of antiretroviral treatment. The patient presented with 1-month history ofneck and dorsal pain and left upper extremitynumbness and weakness. Although the etiology of the cervical spinal cord abscess could not be detected (cryptogenetic ISCA), the patient was successfully managedwith antitoxoplasmosis and antituberculosis treatment. Our findings indicate that an ISCA should be considered in HIV-infected patients with central nervous system signs or symptoms, regardless of CD4 T-cell counts.
\end{abstract}

Keywords: spinal cord abscess, intramedullary abscess, cervical spinal cord, human immunodeficiency virus, magnetic resonance imaging, diffusion-weighted imaging
Volume 9 Issue 3 - 2019

\section{Monique Boukobza, Jean-Pierre Laissy \\ Department of Radiology, Assistance Publique-Hôpitaux de Paris, Bichat Hospital, France}

\begin{abstract}
Correspondence: Monique Boukobza, Department of Radiology, Assistance Publique-Hôpitaux de Paris, Bichat Hospital, 75018, Paris, France, Tel + 336246529 32, Fax+ 33 । 402583 05, Email m.boukobza@orange.fr
\end{abstract}

Received: Novembre 30, 2018 | Published: June 26, 2019

\section{Introduction}

The incidence of spinal infections has increased owing to immunocompromised conditions and IV drug use.

Nonetheless, intramedullary spinal cord abscess (ISCA) is a rare condition especially in the antibiotic ${ }^{1}$ and there are fewer than 150 reported cases in the literature. ${ }^{2}$

We report the magneticresonancei maging (MRI) findings, including the diffusion-weighted imaging (DWI) findings, of ISCA in a HIV-infected 43-year-old male. Multiple infectious organisms include bacteria, fungi, and mycobacteriumhave been reported as etiologic agents. Standard therapy includes surgical drainage and appropriate antibiotic therapy, but a lot of cases have been reported with good outcome after antibiotic treatment alone. In our case, the radiological abnormalities and neurologic deficits resolved after

\section{Case}

A 43-year-old malewith a medical history of HIV infection for 7 years and a breakdown of antiretroviral therapy (ART) for 4years, presented with 1month history of neck and dorsal pain and difficulty walking. Neurological examination was significant for decreased left hand strength (wrist extensor 4/5), and positive left Babinski.

On blood testing at admission the complete blood count, comprehensive metabolic panel, inflammatory markers such as C-reactive protein level were normal. Brain MRI was normal. Spinal cord MRI showed cord enlargement at C4-C5 level and diffuseT2images high signal from medulla to $\mathrm{T} 4$ level. A central area of necrosis hypointense was found on T1-images at C4-C5 level with peripheral enhancement after contrast (Figures 1A-1C). Together, these MRI findings were suggestive of an ISCA. Diffusion weighted images (DWI) showed hyposignal consistent with restricted diffusion on ADC map in the area of the necrotic portion of the cordlesion (Figures 1D\&1E).

Full-body CT-scan was normal. Cerebrospinal fluid (CSF) analysis showed 5 white cells $/ \mathrm{mm}^{3}$ and protein of $530 \mathrm{mg} / \mathrm{L}$. Blood cultures resulted negative for Mycobacteriumtuberculosis, fungi and bacteria. Tests for tuberculosis, toxoplasmosis, cryptococcosis, syphilis, human simplex virus 1 and 2, varicella-zoster virus, hepatitis B virus, cytomegalovirus, Epstein-Barr enterovirus and JC virus were negative. Serological assays for toxoplasmosis, brucellosis, syphilis and Lyme disease in CSF were negative as well.

CD4 T-cell count was at 10 cells $/ \mu \mathrm{L}$ with a plasma viral load of around 611000 copies $/ \mathrm{mL}$. As the patient was HIV-infected withART-breakdown, treatment for toxoplasmosis (sulfadiazine and azithromycin), and tuberculosis (rifampin, ethambutol), was started. Patient's motor weakness gradually improved.

MRI follow-up at 10 days showed animprovedintramedullary T2 hyperintensity and enhancement, but therewas a residualenhancing nodule at the C4-C5 level (Figures 1F \& 1G). At 6months follow-up the neurological examination was unchanged and MRI showed a faint enhancement of the spinal cord at the same level (Figure 1E).
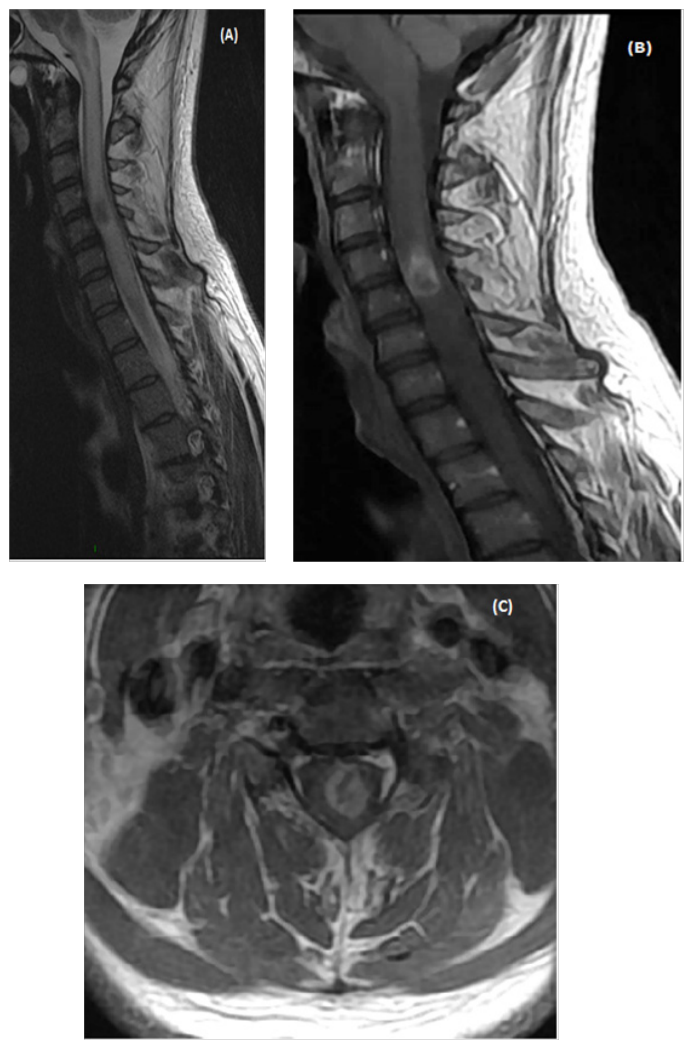

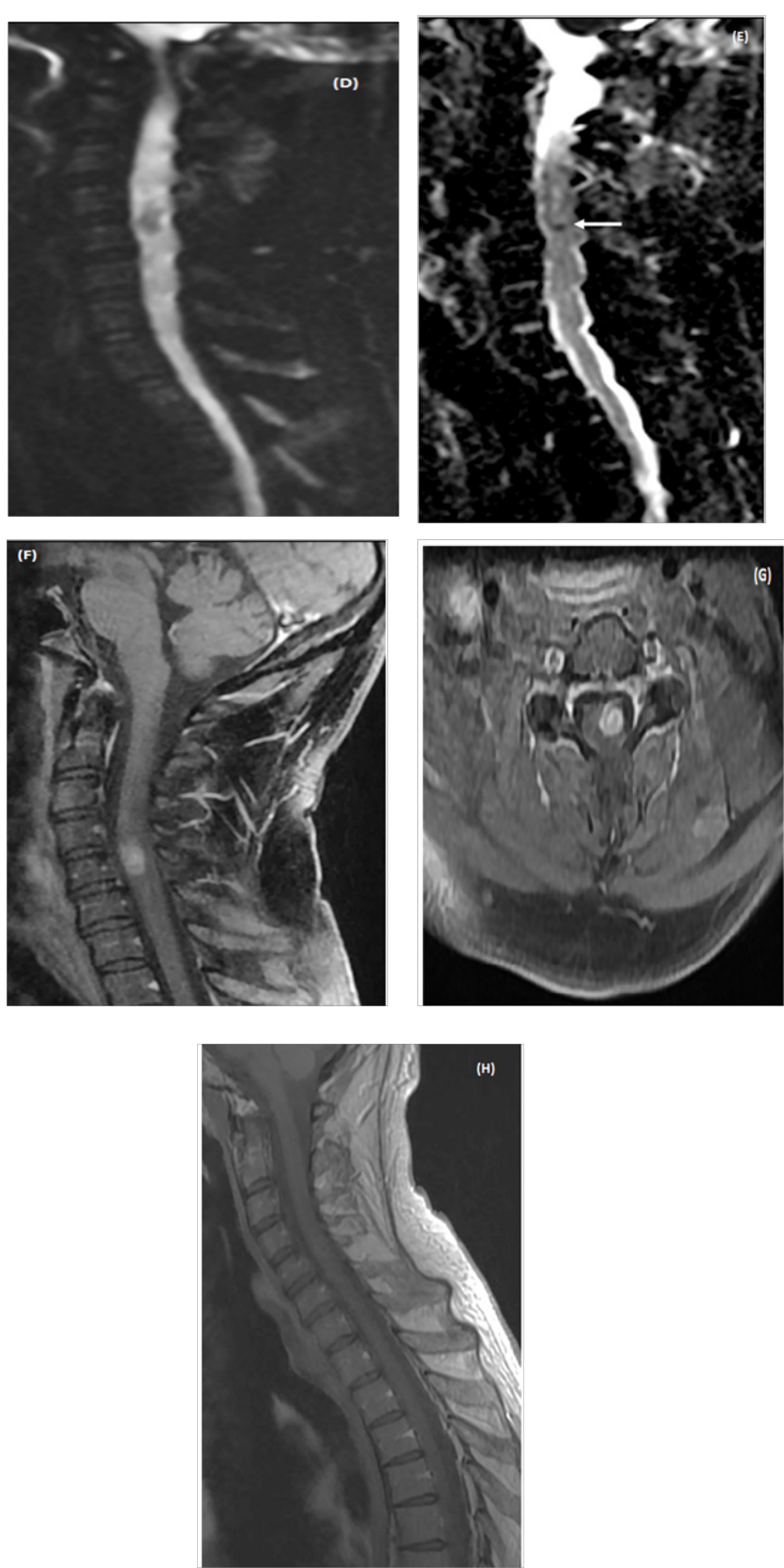

Figure I MRI of an intramedullary cervical spinal cord abscess.

(A) T2-weighted sagittal view of the cervical spine shows intramedullary hyper signal intensity from medulla to T4 level together with expansion of the spinal cord, consistent with edema. Note the central area of necrosis at C4-C5 level. TI-weighted sagittal (B) and axial (C) post-contrast images, showring-enhancement of the lesion at C4-C5 level. The vertebral bodies are intact without disc space infection, epidural collection, or sub dural empyema. (D)The enhancing part of the lesionis slighly hyper intense on sagittal Diffusion-weighted (DW) « focus » sequence (E) Restricted diffusion is visible as a dark dot inside the necrotic area on ADC map (arrow). Follow-up MRI of cervical spine (TI-weighted sagittal (F) and axial (G) post-contrast images performed 14 days after initiation of antibiotic treatment shows partial resolution of the lesion. $\mathrm{h}$-At 6 month-MRI follow-up the lesion has disappeared.

\section{Discussion}

ISCA is a rare condition. The review of the literature by Kurita et al. ${ }^{3}$ found 26 reported cases between 1998 and 2007. Sinha et al in $2013^{2}$ found less than 150 cases reported. The commonest microorganisms responsible for IASC is Staphylococcus followed by Streptococcus although a number of other organisms such as E. coli, Proteus, Listeria, Pseudomonas, Brucella, Histoplasma, Nocardia, Toxoplasma and Mycobacterium have been implicated. ${ }^{4,5}$ There are few reports about Toxoplaamagondii in $\mathrm{HIV}+$ patients in the literature and PCR onCSFis not sensitive enough as a diagnostic tool. ${ }^{6}$

Our patient was considered as a typical case of intramedullary spinal cord abscess (IASC) despite the negative CSF and blood cultures for 2 reasons: the spinal cord lesion had the MRI characteristics of ISCA and the rapid regression of the lesion on MRI control after probabilistic anti-toxoplasmosis and anti-tuberculosis treatment was initiated.

In the present case, as global biological analysis and radiological investigations were negative, the mechanism of infection remains cryptogenic. The ISCA developed while the CD4 T-cell count was very low. Patient's HIV was the only predisposing factor. The IASC usually occurs in patients with diabetes mellitus, or intravenous drug abuse or immunosuppression as in the current case, the HIVinfected patient being non-controlled as evidenced by his CD4 count and viral load.

Most of the time, it occurs as a consequence of a hematogenous spread from an extra spinal infected focus: respiratory tract (the most frequent source), cardiac (bacterial endocarditis), skeletal, genitourinary, gastrointestinal or cutaneous. Contiguous spreads from a dermal sinus, dermoid cyst with dermal sinus tract are predisposing factors in pediatric ISCA. Extension from adjacent structures infection as bacterial meningitis, vertebralosteomyelitis ${ }^{7}$ or spondylodiskitis. ${ }^{8}$ Other cases are associated with direct trauma and neurosurgical intervention. The primary source of infection could not be found in $30 \%$ of cases and they are called cryptogenic ISCA. ${ }^{1}$ In most reported cases, examination of CSF showed normal values or non-specific changes as elevated protein. Rarely, signs of inflammation or pus are found in CSF.

The symptoms and signs of IASC are often the same than those of epidural abscess. However, in most cases they are nonspecific and they do not allow a final diagnosis. Symptoms include pain $(60 \%)$, motor and sensory deficits (68\%), urinary incontinence $(56 \%)$, fever $(40 \%)$ and spine pain and/or stiffness $(12 \%))^{2,3}$

Initial MRI demonstrates intramedullary hyperintensity on T2sequence and poorly defined marginal enhancement on T1- postcontrast sequence. On MRI follow-up, IASC present a ring- like enhancement with central low signal intensity on post-contrast T1images.These MRI features are similar to those of brainabscesses. ${ }^{9}$ On further MRI follow-up, the enhancement resolve under antibiotic therapy.

DWI can help diagnose IASC. However, it is not routinely performed. Usually, the non-enhancing necrotic part of the lesion appears hyper-intense on DWI with decreased apparent diffusion coefficient (ADC) on the ADC map. ${ }^{7,10-12}$ However, the hyperintensity on DWI and ADC-restriction may vary in the abscesscavity according to the viscosity and age of the abscess, as demonstrated in the current 
case. The reported cases of DWI in IASC did not concern spinal cord toxoplasmosis. Whatever, the sequential imaging findings, showing regression of the lesion as in our case, aids in the differentiation of cord infection from other intramedullary lesions.

The aspect of intramedullary ring-enhancing mass is a nonspecific feature. For adequate medical and surgical treatment, it is crucial to differentiate ISCA from necrotic tumors, including primary (necrotic glioma) or secondary spinal cord tumors (necrotic metastases) as resolving hematoma, infarction and demyelinating disease. Necrotic spinal cord tumors present with low signal intensity on DWI and show increased values of ADC. For these reasons, DWI may be very helpful for the diagnosis: The pus in the abscess cavity presents a hyperintensity on DWI and diffusion restriction on ADC map, due to its high viscosity. In most reported cases, patients underwent surgical abscess drainage together with antibiotic treatment. ${ }^{2,7,11,13,14}$ In HIV patients the biopsy must be necessary ${ }^{15}$ and in cases ISCA resolve with antibiotic treatment alone. ${ }^{3}$ We chose medical treatment as single approach because of the fast and satisfactory initial response of both the clinical and the imaging findings.

\section{Conclusion}

As ISCA is a rare infection of the CNS, a high index of suspicion, early recognition of ISCA and immediate aggressive medical and/or surgical treatment are essential to prevent significant morbidity and mortality associated with rare infection.

Our case shows that HIV-infected patients with ART break down may develop crypto genetic ISCA. The use of diffusion weighted imaging of the spinal cord can provide an accurate diagnosis and serial evaluation with MRI is useful to monitor treatment

\section{Acknowledgments}

None.

\section{Conflicts of interest}

Author declares there are no conflicts of interest.

\section{References}

1. Chan CT, Gold WL. Intramedullaryabscess of the spinal cord in the antibioticera: Clinicalfeatures, microbialetiologies, trends in pathogenesis, and outcomes. Clin Infect Dis. 1998;27(3):619-626.

2. Sinha P, Parekh T, Pal D. Intramedullary abscess of the upper cervical spinal cord. Unusual presentation and dilemmas of management: Case report. Clin Neurol Neurosurg. 2013;115 (9):1845-1850.
3. Kurita N, Sakurai Y, Taniguchi M, Terao T, Takahashi H, Mannen Tet al. Intramedullary spinal cord abscess treated with antibiotic therapy-case report and review. Neurol Med Chir (Tokyo). 2009;49(6):262-268.

4. Bartels Ronald HMA, GoneraEdG, van der Spek Johannes AN, et al. Intramedullary spinal cord abscess. Spine. 1995;20(10):1199-1204.

5. Peeters I, Casselman JW, Vandecasteele SJ, et al. Multiple nocardialabscesses of the brainstem and spinal cord diagnosedafter an open biopsythrough a cervical partial central corpectomy: case report. $J$ Neurosurg Spine. 2015;23(3):290-293.

6. Pittner Y, Dufour JF, David G, et al. Spinal cord toxoplasmosis in HIV infection. Med Mal Infect. 2009;39(6):401-405.

7. Takebe N, Iwasaki K, Hashikata $\mathrm{H}$, et al. Intramedullary spinal cord abscess and subsequent granuloma formation: a rare complication of vertebral osteomyelitis detected by diffusion-weighted magnetic resonance imaging. Neurosurg Focus. 2014;37(2):E12.

8. Derkinderen P, Duval X, Bruneel F, et al. Regnier B. Intramedullary spinal cord abscess associated with cervical spondylodiscitis and epidural abscess. Scand J Infect Dis. 1998;30(6):618-619.

9. Murphy KJ, Brunberg JA, Quint DJ, et al. Spinal Cord Infection: Myelitis and Abscess Formation. AJNR Am J Neuroradiol. 1998;19(2):341-348.

10. Dörflinger-Hejlek E, Kirsch EC, Reiter H, et al. Diffusion-weighted MR imaging of intramedullary spinal cord abscess. AJNR Am J Neuroradiol. 2010;31(9):1651-1652.

11. Roh JE, Lee SY, Cha SH, et al. Sequential Magnetic Resonance Imaging Finding of Intramedullary Spinal Cord Abscess including Diffusion Weighted Image: a Case Report. Korean J Radiol. 2011;12(2):241-246.

12. Moritani T, Kim J, Capizzano AA, et al. Pyogenic and non-pyogenic spinal infections: emphasis on diffusion-weighted imaging for the detection of abscesses and pus collections. Br J Radiol. 2014;87(1041):20140011.

13. Hood B, Wolfe SQ, Trivedi RA, et al. Intramedullary abscess of the cervical spinal cord in an otherwise healthy man. World Neurosurg. 2011;76:361. e15-361.e19.

14. Bakhsheshian J, Kim PE, Attenello FJ. Intramedullary Cervical Spinal Cord Abscess. World Neurosurg. 2017;106:1049.e1-1049.e2.

15. García-García C, Castillo-Álvarez F, Azcona-Gutiérrez JM, et al. Spinal cord toxoplasmosis in human immunodeficiency virus infection/acquired immunodeficiency syndrome. Infect Dis (Lond). 2015;47(5):277-282. 